Gynäkologe 2019· 52:181-182

https://doi.org/10.1007/s00129-019-4390-1

(c) Springer Medizin Verlag GmbH, ein Teil von Springer Nature 2019

CrossMark

\title{
Th. Dimpfl
}

Frauenklinik, Klinikum Kassel, Kassel, Deutschland

\section{Urogynäkologie: vom Stiefkind zum Hauptdarsteller}

Angesichts der zunehmenden Spezia-

Die Zusammensetzung der Bevölkerung hat sich in den letzten 20-40 Jahren drastisch verändert. Vom Statistischen Bundesamt publizierte Zahlen zeigen in 1990 einen Bevölkerungsanteil älter als 65 Jahre von $15 \%$ und in 2005 einen von $19 \%$. Prognostiziert wird für 2020 ein Anteil von $23 \%$.

In einer der weltweit größten epidemiologischen Erhebungen im Rahmen der norwegischen EPICONT-Studie, in der 28.000 Frauen zur Harninkontinenz befragt wurden, gaben $25 \%$ der Frauen unfreiwilligen Harnverlust an. Die Häufigkeit stieg mit wachsendem Alter.

Die Prävalenz des Descensus genitalis wird in einer schwedischen Studie mit $31 \%$ in einer Subgruppe von Frauen zwischen 20 und 59 Jahren angegeben. Die Prävalenz des Deszensus bei Frauen zwischen 50 und 79 Jahren steigt auf bis zu $41 \%$. Das lebenslange Risiko, wegen eines Genitaldeszensus oder einer Inkontinenz operiert zu werden, beläuft sich auf 11-19\%, das Risiko für eine Rezidivoperation liegt sogar bei $29 \%$.

Durch die hohe Prävalenz von Kontinenz- und Beckenbodenproblemen bei Frauen ergibt sich daher ein enormer Versorgungsauftrag und eine vielversprechende Perspektive für die Subspezialität Urogynäkologie.

Die Ursachen für Inkontinenz und Deszensus sind in aller Regel multifaktoriell. Eine subtile und umfassende Diagnostik ist die Grundlage für jede erfolgreiche Therapie, ob konservativ oder operativ. Diese aufwendige Diagnostik wird im aktuellen Vergütungssystem nur unzureichend abgebildet. Die Behandlungsmethoden für die verschiedenen Formen der Inkontinenz und für den Descensus genitalis unterliegen einem fortlaufenden Wandel. lisierung wurden 2003 auf dem Gebiet der Urogynäkologie erstmals deutschsprachige Leitlinien in Zusammenarbeit mit Kollegen aus Österreich und der Schweiz verfasst. Dieses Konzept war das erste dieser Art und später wegweisend für viele andere Leitlinien. Diese wurden regelmäßig und je nach Bedarf aktualisiert, erweitert und interdisziplinär gestaltet. Die Leitlinien beruhen auf wissenschaftlichen Erkenntnissen wie auf in der Praxis bewährten Verfahren und berücksichtigen auch ökonomische Aspekte.

Meilensteine in der operativen Behandlung in der Urogynäkologie waren in 1996 die Einführung des TVT („tension-free vaginal tape“) und in $2001 \mathrm{der}$ transobturatorische Zugang.

\section{》) In spezialisierten Zentren werden urogynäkologische Interventionen meist minimal- invasiv durchgeführt}

Im Jahr 2003 kam es zur Einführung der vaginalen Netze. In 2009 und 2011 gab die FDA (Food and Drug Administration) Warnungen vor der Verwendung der vaginalen Netze heraus. Als Konsequenz kündigten in 2012 namhafte Hersteller weltweit (z.B. Ethicon, AMS, Bard) ihren Rückzug aus diesem Markt an.

In 2017 publizierten Morling et al. im Lancet eine 20-jährige Untersuchung zum Einsatz von Netzen und Bändern in Schottland in der Inkontinenz- und Deszensusbehandlung, die weltweit Beachtung fand: Durch diese Studie nahm die Verunsicherung bei Ärzten und Patientinnen weiter $\mathrm{zu}$. In der Folge publizierten
Experten aus ganz Europa mit deutscher Beteiligung ein Konsensus-Statement der EUGA (European Urogynaecology Association), in dem Empfehlungen zur Anwendung gegeben werden.

Die operative Therapie hat sich in den letzten Jahren die Fortschritte und Entwicklungen der minimal-invasiven Chirurgie zunutze gemacht. So werden heute in spezialisierten Zentren mehr oder weniger alle urogynäkologischen Eingriffe minimal-invasiv durchgeführt. Eine weitere Entwicklung ist die kritische Frage der über viele Jahrzehnte praktizierten Mitentfernung des Uterus im Rahmen von Deszensuseingriffen. Es gibt mittlerweile auch gute Daten aus Deutschland, die zeigen, dass der Uteruserhalt die Langzeitergebnisse eher verbessert.

In Analogie zur Versorgung von $\mathrm{Pa}$ tientinnen mit Brustkrebs in zertifizierten Brustkrebszentren ist eine ganzheitliche Betreuung von Frauen mit Beckenbodenproblemen in zertifizierten, interdisziplinären Beckenbodenzentren wünschenswert und anzustreben. Ein wichtiger, zukunftsweisender Aspekt ist die Aus- und Weiterbildung des Nachwuchses sowie die Harmonisierung mit den europäischen Vorgaben und Gegebenheiten (Eastern Cooperative Oncology Group, ECOG). Dafür ist die Etablierung des Schwerpunktes Urogynäkologie nach europäischem und Schweizer Modell unverzichtbar.

\section{》) Der Bedarf an gut ausgebildeten Urogynäkologen wird weiter steigen}

Für die Subspezialität Urogynäkologie ergeben sich vielversprechende Perspektiven und die Nachfrage nach gut aus- 
gebildeten Urogynäkologen wird weiter steigen.

Wir wünschen Ihnen beim Studium dieses Heftes mit dem Schwerpunkt Urogynäkologie einen hohen Wissenszugewinn und Antworten auf Ihre Fragen.

Für die Autoren N. Alardeir, K. Beilecke, M. Deniz, A.-K. von Hobe, U. Peschers, G. Naumann, C. Reisenauer und C. Schiffner,

Ihr

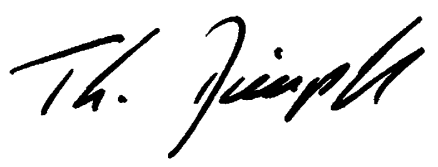

Prof. Dr. med. T. Dimpfl

\section{Korrespondenzadresse}

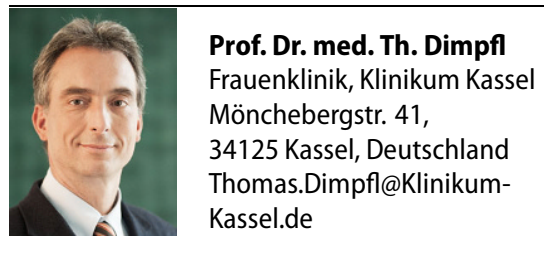

Interessenkonflikt. T. Dimpfl gibt an, dass kein Interessenkonflikt besteht.

\section{A. Platte \\ Das Ereignis der Geburt}

Medizinisches Wissen und Deutung des Geburtsaktes vom ausgehenden 18. bis zur Mitte des 19. Jahrhunderts

Bern, Schweiz: Peter Lang International Academic Publishers 2018, 1. Auflage, 270 S., (ISBN: 978-3631747261), Gebunden 49,95 EUR

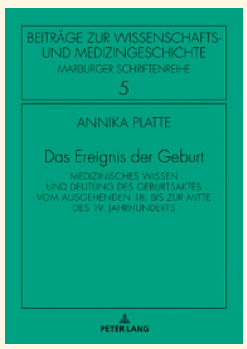

Annika Platte, Frauenärztin, tätig in Karlstadt, Schweden, legt ein medizinhistorisches Buch vor, welches detailliert und mit zahlreichen Quellenangaben versehen den Stand der Ansichten und des Wissens nachzeichnet, den man im 18. und 19.Jh. über die Entstehung menschlichen Lebens, Schwangerschaft und Geburt besaß.

Es ist erst 200 Jahre her, dass man darüber diskutierte, gelegentlich auch erbittert stritt, ob der Mensch präformiert, d.h. embryonal schon in der Anlage fertig geschaffen sei (Albrecht von Haller), oder epigenetisch, d.h. infolge einer den Körper gestaltenden, ausbildenden (und später auch regenerierenden) Kraft entstehe (Caspar Friedrich Wolff). Später standen Untersuchungen zur Atmung (Paul Zweifel) und Ernährung (William Preyer) des Feten im Vordergrund. Die Erkenntnis der Funktion von Plazenta und Fruchtwasser gewannen wissenschaftliches Interesse. Bezüglich der Auslösung der Geburt herrschten vom 17. bis zur Mitte des 18.Jh. verschiedene kontroverse Ansichten vor: Reifung der Plazenta, Dehnungseinflüsse auf die Uteruswand durch den wachsenden Fetus, muskuläre Reize, Hungerreaktion des Feten. Die muskuläre Beschaffenheit des Uterus stand danach im Mittelpunkt, als Alfred Kölliker die Existenz glatter neben der bekannten quergestreiften Muskulatur nachgewiesen hatte (1847). Von nun an konzentrierte sich die wissenschaftliche Debatte um das Zusammenwirken der muskulär-uterinen Geburtsphysiologie und der fötalen Autonomie.

Führend in der Schaffung eines wissenschaftlich begründeten Bildes wurden nun Friedrich Benjamin Osiander (1759-1822), Johann Christian Jörg (1779-1856), Carl Gustav Carus (1789-1869), Dietrich Wilhelm Heinrich
Busch (1788-1858) und Friedrich Wilhelm Johann Ignaz Scanzoni (1821-1891). Sie werden mit Biographien und ihren Beiträgen ausführlich dargestellt. Für die Geburtshilfe der Romantik, vertreten durch die genannten Forscher, bestand noch ein enges Verhältnis zur Naturphilosophie. Die Zusammenhänge mit der endokrinen Physiologie - der Begriff Hormone wurde erst 1905 von Ernest Starling (1866-1927) geprägt - verwandelte sich das Wissen um die Auslösung der Geburt grundlegend bis hin zu unseren heutigen Kenntnissen. Es ist das große Verdienst der Autorin, den langwierigen Weg nachgezeichnet zu haben, den unsere Kenntnisse bis heute genommen haben.

H. Ludwig (Basel) 